\title{
Local and Remote Cooperation With Virtual and Robotic Agents: A P300 BCl Study in Healthy and People Living With Spinal Cord Injury
}

\author{
Emmanuele Tidoni, Mohammad Abu-Alqumsan, Member, IEEE, Daniele Leonardis, Member, IEEE, \\ Christoph Kapeller, Gabriele Fusco, Cristoph Guger, Member, IEEE, Cristoph Hintermüller, \\ Angelika Peer, Member, IEEE, Antonio Frisoli, Member, IEEE, Franco Tecchia, \\ Massimo Bergamasco, Member, IEEE, and Salvatore Maria Aglioti
}

\begin{abstract}
The development of technological applications that allow people to control and embody external devices within social interaction settings represents a major goal for current and future brain-computer interface $(\mathrm{BCl})$ systems. Prior research has suggested that embodied systems may ameliorate $\mathrm{BCl}$ end-user's experience and accuracy in controlling external devices. Along these lines, we developed an immersive $\mathrm{P} 300$-based $\mathrm{BCl}$ application with a head-mounted display for virtual-local and robotic-remote social interactions and explored in a group of healthy participants the role of proprioceptive feedback in the control of a virtual surrogate (Study 1). Moreover, we compared the performance of a small group of people with spinal cord injury (SCl) to a control group of healthy subjects during virtual and robotic social interactions (Study 2), where both groups received a proprioceptive stimulation. Our attempt to combine immersive environments, $\mathrm{BCl}$ technologies and neuroscience of body ownership suggests that providing realistic multisensory feedback still represents a challenge. Results have shown that healthy and people living with $\mathrm{SCl}$ used the $\mathrm{BCl}$ within the immersive scenarios with good levels of performance (as indexed by task accuracy, optimizations calls and Information Transfer Rate) and per-
\end{abstract}

Manuscript received March 29, 2016; revised August 11, 2016 and October 24, 2016; accepted November 3, 2016. Date of publication December 23, 2016; date of current version September 2, 2017. The research was funded by the EU Information and Communication Technologies Grant (VERE project, FP7-ICT-2009-5, Prot. Num. 257695) and the Italian Ministry of Health (RF-2010-2312912) to SMA. ET was supported by BIAL Foundation (2014/150). E. Tidoni, M. Abu-Alqumsan, and $\mathrm{D}$. Leonardis contributed equally.

E. Tidoni, G. Fusco, and S. M. Aglioti are with the Department of Psychology, University of Rome "La Sapienza", Rome 00185, Italy and also with Fondazione Santa Lucia IRCCS, Rome, Italy (e-mail: mmanuele.tidoni@uniroma1.it; gabriele.fusco@uniroma1.it; salvatoremaria.aglioti@uniroma1.it).

M. Abu-Alqumsan is with the Chair of Automatic Control Engineering, Technical University of Munich, TUM, Munich, Germany (e-mail: moh.marwan@Isr.ei.tum.de).

D. Leonardis, A. Frisoli, F. Tecchia, and M. Bergamasco are with Percro Laboratory, Scuola Superiore Sant'Anna, Pisa, Italy (e-mail: d.leonardis@sssup.it; a.frisoli@sssup.it; f.tecchia@sssup.it; bergamasco@sssup.it).

C. Kapeller, C. Guger, and C. Hintermüller are with Guger Technologies OG, 8020 Graz, Austria and also with g.tec medical engineering GmbH, Schiedlberg, Austria (e-mail: kapeller@gtec.at; guger@gtec.at; hintermueller@gtec.at).

A. Peer is with the Bristol Robotics Laboratory, University of the West of England, Bristol, Bristol, U.K. (e-mail: angelika.peer@brl.ac.uk).

This paper has supplementary downloadable material available at http://ieeexplore.ieee.org., provided by the author.

Digital Object Identifier 10.1109/TNSRE.2016.2626391 ceived control of the surrogates. Proprioceptive feedback did not contribute to alter performance measures and body ownership sensations. Further studies are necessary to test whether sensorimotor experience represents an opportunity to improve the use of future embodied $\mathrm{BCl}$ applications.

Index Terms-Body illusions - tendon vibration, brain-computer interface (BCI) $\mathrm{P} 300$, spinal cord injury, teleoperation, virtual reality.

\section{INTRODUCTION}

B RAIN-COMPUTER interfaces (BCIs) provide a nonmuscular communication channel by capturing through electroencephalography (EEG) the electrical signals generated in the brain and translating them into actions that reflect user's intentions [1], [2].

Moving from the laboratory to the real world represents a big challenge for current and future BCI-based applications [3]-[5]. Such challenge is more pronounced for immersive applications that aim at enabling human-human interactions, which are typically characterized by the integration of different sensory channels (e.g., visual, tactile) and dynamic exchanges of information between multiple agents. While in real life we seamlessly interact with the environment and other humans using our own body, during remotelycontrolled interactions the user's body is substituted by a virtual or robotic agent. Systems, whereby users can be immersed within a virtual reality (VR) or remote environment through a virtual or robotic surrogate [6] controlled by a BCI, may allow to explore thought-based social interactions [7], [8] within safe settings. Former research in immersive VR systems has shown that a person can transfer his/her body into a virtual surrogate [9] and feel to "be there", in the place where the avatar is acting [10], [11].

Recent studies highlighted that motivation may improve performance in motor-imagery-based BCIs [12], [13] and may increase the amplitude of $\mathrm{P} 300$ potentials [14] (a positive deflection is detected $300 \mathrm{~ms}$ after the presentation of the attended stimulus). Similarly, it was suggested that embodiment feeling may increase motor imagery BCI control [15] and error-related EEG signals [16], [17]. On the other hand, the congruency among multiple afferents is fundamental to embody an external object [18] and the ownership feeling towards a virtual or robotic agent can be modulated, for 
TABLE I

CLINICAL AND DEMOGRAPHICAL INFORMATION OF PEOPLE LIVING WITH SCI

\begin{tabular}{|ccccccc|}
\hline Case & $\begin{array}{c}\text { Age } \\
\text { (Years) }\end{array}$ & $\begin{array}{c}\text { Months } \\
\text { since injury }\end{array}$ & Gender & $\begin{array}{c}\text { Lesion } \\
\text { level (AIS) }\end{array}$ & Etiology & $\begin{array}{c}\text { Illusory } \\
\text { movement }\end{array}$ \\
\hline Pt1 & 22 & 94 & M & C4-C5 (B) & Traumatic & Yes \\
Pt2 & 29 & 84 & M & C6 (D) & Traumatic & Yes \\
Pt3 & 31 & 88 & M & C4 (A) & Traumatic & No \\
\hline
\end{tabular}

instance, through synchronous visuo-tactile stimulations [19] or through the match between the BCI commands issued by the users and the visual feedback [20], [21] provided to them.

In line with these findings, we considered a BCI-based social interaction scenario in which proprioceptive stimulation and visual feedback were provided, aiming at recreating a sensorimotor experience closer to what can be observed in real human-human interactions. We combined immersive virtuallocal and robotic-remote scenarios (by means of a headmounted display, HMD) with a BCI application, whereby participants cooperated with a virtual and a real partner, respectively, in a board game that required solving simple mathematical operations. We designed the application for both healthy and spinal cord injured (SCI) participants providing, in addition to the visual feedback, tendon proprioceptive stimulation that is known to induce the illusory experience of arm movement [22] as sensory feedback. We qualitatively checked the user experience (UE) with a questionnaire and quantitatively assessed performance as measured by task accuracy (the percentage of successful trials; see Study1 Section A), optimization calls (see Materials and Methods Section C) and information transfer rate (ITR; see Study1 Section A). This way, we explored the role of proprioceptive feedback in healthy and people living with SCI during a BCI-based social interaction task. In particular, we assessed in healthy participants whether different proprioceptive stimulation affected embodiment feeling and performance measures within a VR immersive environment (Study 1). Moreover, we compared a sample of tetraplegic patients (Pts) with a control group of healthy subjects in a robotic and virtual scenario (Study 2). We hypothesized higher embodiment and performance levels during illusory movement experience compared to non-illusory proprioceptive stimulation and comparable performance between healthy and people living with SCI.

\section{Materials and Methods}

\section{A. Participants}

A total of 21 participants were enrolled in the two studies. Eight healthy participants ( 4 males; mean \pm std., $27.00 \pm 3.50$, range 22-32) were recruited for Study 1 . Ten healthy participants (6 males; $29.33 \pm 2.87$, range 24-32) and three participants with a SCI $(28.00 \pm 5.19$, range $22-31$, see Table I for details) were recruited for Study 2. Patient 3 (Pt3) was previously tested in a rubber hand paradigm [23]. All participants were right-handed, had normal or correctedto-normal visual acuity in both eyes, and were naïve as to the purposes of the study. The experimental protocol was

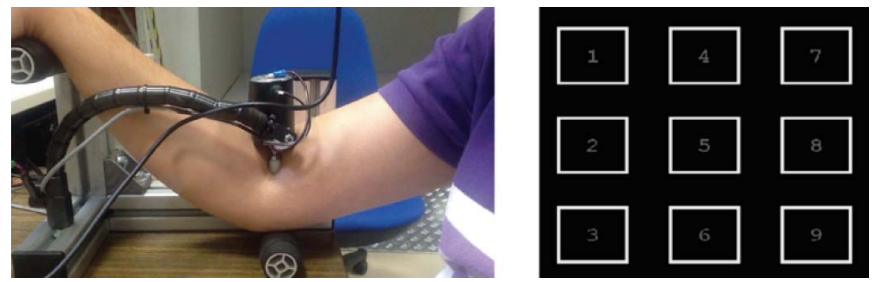

Fig. 1. (Left) The proprioceptive device supported participants' right arm and stimulated the tendon biceps brachii using a hemispherical tactor (15 mm diameter), mounted at the tip of the actuated moving shaft. (Right) P300 mask used during the $\mathrm{BCl}$ training and accuracy assessment (see Section II-D).

approved by the ethics committee of the Fondazione Santa Lucia and was carried out in accordance with the ethical standards of the 1964 Declaration of Helsinki. All participants gave their written informed consent to take part in the study. No discomfort or adverse effects were reported or noticed at any time during the experiment.

\section{B. Hardware and Software Integration}

The experimental setup was composed of the following modules: a proprioceptive stimulator device, a P300-based BCI, a computer graphic virtual environment and a teleoperated robot. The game logic and the interface among the different modules were developed using XVR (XVR media, [24], [25], [26]). More details on each of these modules are provided in the next subsections.

Proprioceptive stimulator device. Proprioceptive stimulation was provided through a device [27] specifically designed to evoke an illusory extension of the forearm induced by tendon vibration (TV; see [28] for details; Fig. 1 left). In particular, in Study 1 we stimulated the right tendon of the biceps brachii [22] to elicit the illusion of elbow downward extension (proprioceptive stimulation with illusion, MovI + ), and the skin surface over the bone nearby the biceps tendon [29] to generate a tactile vibratory sensation without perception of illusory movements (proprioceptive stimulation without illusion, MovI-). In Study 2 we used only the MovI+ stimulation.

P300-based BCI. EEG signals were acquired at $256 \mathrm{~Hz}$ by means of a g.USBamp (24 Bit biosignal amplification unit, g.tec Medical Engineering $\mathrm{GmbH}$, Austria). Eight $\mathrm{Ag} / \mathrm{AgCl}$ active electrodes were placed at the $\mathrm{Fz}, \mathrm{Cz}, \mathrm{P} 3, \mathrm{Pz}, \mathrm{P} 4, \mathrm{PO}$, $\mathrm{Oz}$ and $\mathrm{PO} 8$ positions of the extended international 10-20 EEG system. Electrodes were referenced to the right earlobe and the ground electrode was positioned at FPz.

The multi-channel EEG data was then bandpass-filtered in the range $(0.5-30 \mathrm{~Hz})$, notch-filtered at $50 \mathrm{~Hz}$ and downsampled to $64 \mathrm{~Hz}$ [30].

During experiments, we used a $3 \times 3$ P300 board mask (Fig. 1 -upper right) in row-column flashing mode, where all rows and columns were repetitively flashed in random order several times before a decision about user's intention was made. Flash-time (the time, in which a row/column is highlighted on the screen) and dark-time (the time between two consecutive flashes) were respectively set at $133.34 \mathrm{~ms}$ and $83.34 \mathrm{~ms}$ for a $60 \mathrm{~Hz}$ HMD. To ensure visual stimulation with high contrast, we inverted the color of each pixel in 


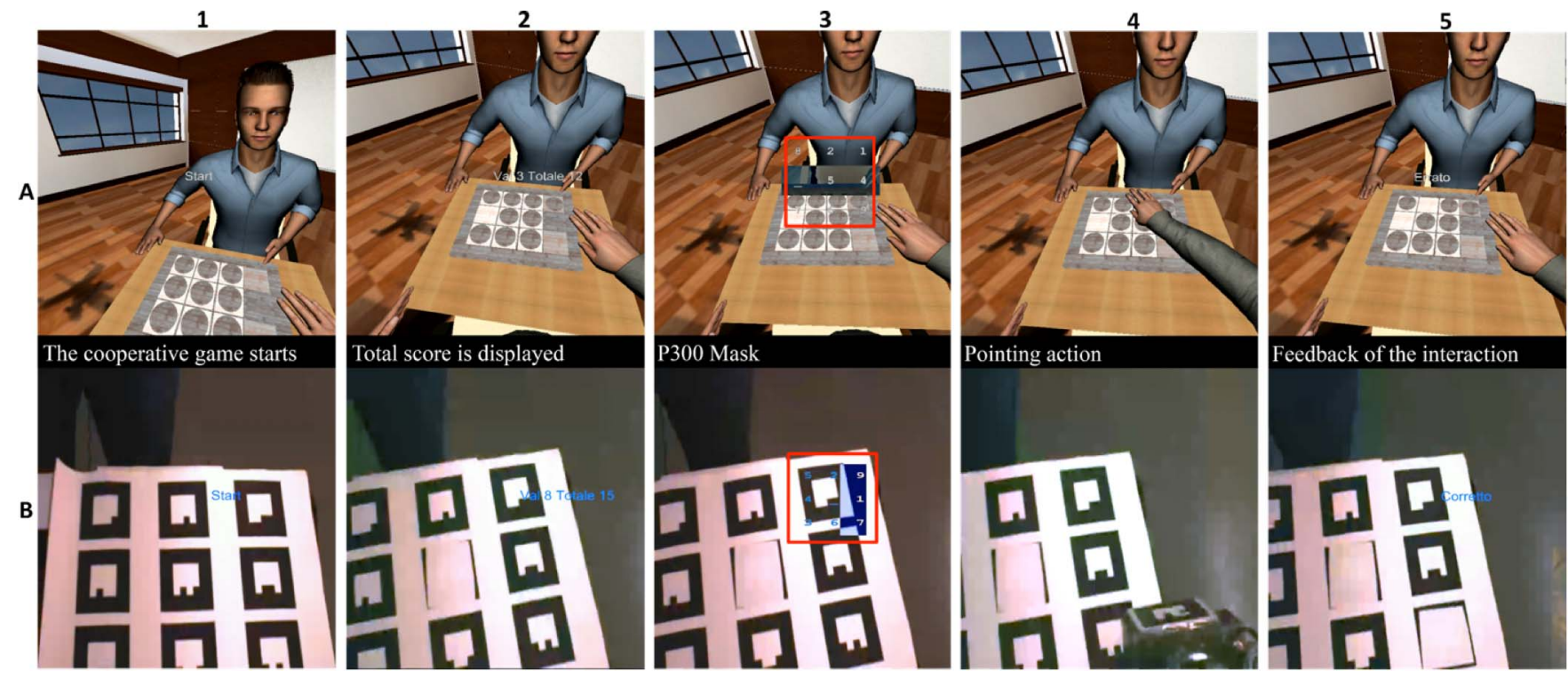

Fig. 2. The BCl-user (BP) observed the virtual/real partner (PP) from a first person perspective The wide field of view noticeable in the screenshots was compensated through the immersive HMD. The figure describes the key events of a single trial (see Section II-C). The P300 mask is highlighted by a red square for illustration purposes.

the scene that was visible under the flashing BCI control elements. A screen overlay control interface (SOCI) [31] was used to perform the P300-based visual stimulation of the BCI in synchrony with the visual feedback provided in the virtual and robotic scenarios.

EEG epochs of length $800 \mathrm{~ms}$ were extracted from the preprocessed EEG data about the onset of each flash, i.e., $100 \mathrm{~ms}$ pre-stimulus (baseline) and $700 \mathrm{~ms}$ post-stimulus. The post-stimulus data were corrected for the baseline and down-sampled by a factor of 3. Data from all electrodes was then concatenated to form feature vectors of length $120(8 * \operatorname{ceil}[0.7 * 64 / 3])$ as the input to the $\mathrm{P} 300$ linear discriminant analysis (LDA classifier.

\section{The Cooperative Game}

Nine tokens were arranged in a $3 \times 3$ grid placed on top of a table (Fig. 2-A1 and B1) and two players, the BCI-user (BP) and his partner (PP), were supposed to cooperate in a mathematical game. Each token corresponded to a number (range 1-9) and the BP and PP had to clear the table by matching tokens that sum up to a given target-score (TS). The PP was blind about the values of the tokens, while the BP was not. Participants performed a total of 12 trials divided into three runs of 4 trials each, as four trials are sufficient to clear the board.

At the beginning of each game run, all the tokens were available on the tabletop and randomly assigned values between 1 and 9 (Fig. 2-A1 and B1). The PP made the first movement by removing a token of own choice from those available. The value of the cleared token became the first addend of the mathematical addition operation (e.g., PP removes a token with value "3"). Then, the BP was informed about both the first addend's value and the TS through a text message that appeared on the HMD display (Fig. 2-A2 and B2; e.g., TS equals to "12" thus, a text message "value: 3 Total: 12" is displayed on the HMD's screen). At this point, the BCI mask for P300 visual stimulation was displayed overlaying the game board (virtual or real). The BP cooperates with the PP by selecting, via the P300-based interface, the token that corresponds to the value which correctly solves the mathematical problem (e.g., "9").

Upon the completion of BCI-selections (Fig. 2-A3 and B3), the surrogate, both in the virtual and the robotic condition (Fig. 2-A4 and B4), performed a pointing action towards the selected token and a proprioceptive stimulation at $60 \mathrm{~Hz}$ and $4.2 \mathrm{~N}$ was provided to the subject's arm. Following the pointing action, the PP removed the pointed-at token and a text message informed the BP about the success or failure of the game trial ("Correct" or "Wrong" respectively; Fig. 2-A5 and B5).

Importantly, the BCI visual interface adapted to the cleared tokens by clearing the corresponding cells in the P300 mask. In this way, the BP could focus his/her attention on the remaining cells/tokens with less distraction. Moreover, upon the selection of an empty cell (a cell previously cleared from the BCI-mask after the corresponding token was removed), a new BCI-selection phase started automatically and lasted until an available token was selected. Throughout the manuscript, we refer to these new BCI-selections as optimization calls (OCs). OCs reduced the total number of wrongly committed actions and increased the overall accuracy by avoiding the selection of unavailable tokens (e.g., tokens already chosen and removed in previous trials). Hereby, OCs indirectly provide information about the participants' need of software interventions to complete the assigned tasks.

Users viewed the virtual/physical environment and the BCI stimuli with the help of an Oculus-DK1 HMD (Oculus VR, USA), that is equipped with an inertial and compass sensor for tracking head orientations and is characterized by a $110^{\circ}$ field-of-view (diagonal FOV), a resolution 


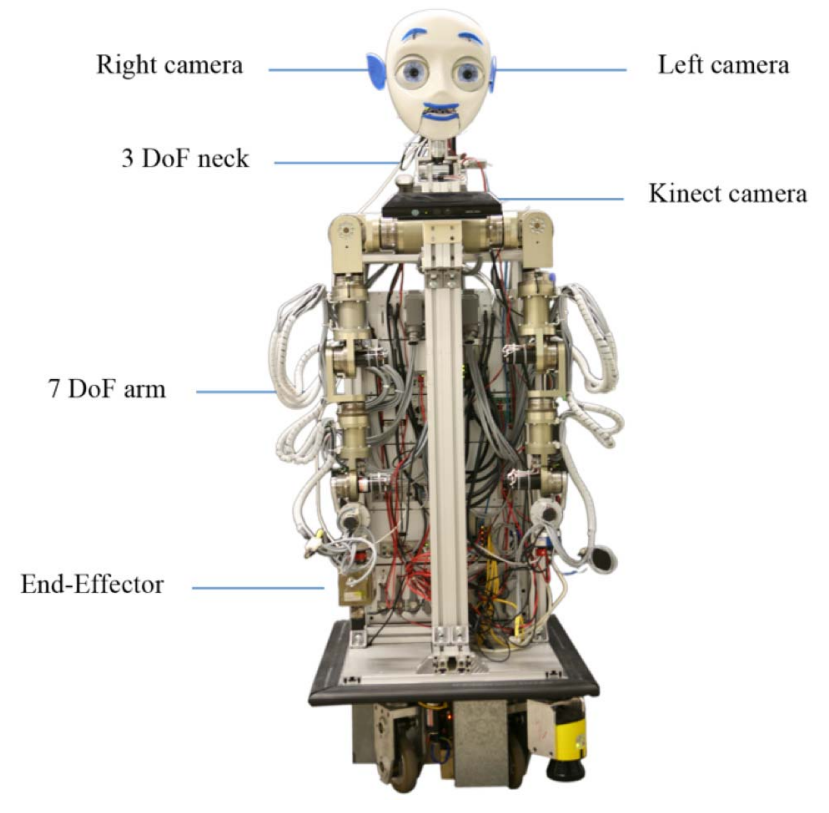

Fig. 3. Teleoperated robot used in the robotic condition. The robot is located at the Chair of Automatic Control Engineering, Technical University of Munich, Munich, Germany.

of $1280 \times 800(16: 10$ aspect ratio, $640 \times 800$ per eye $)$ and $60 \mathrm{~Hz}$ refresh rate.

Virtual scenario. The whole gaming scenario was reconstructed as a 3D-model in computer graphics, and rendered in real time through XVR. The VR scenery was viewed in stereoscopy through the HMD from a first person perspective (1PP), where the BP was immersed into a virtual room and embodied into a virtual avatar. Computer graphic models of the BP and PP avatars were implemented by the HALCA library [32], providing highly detailed and realistic human characters. The real-time tracking/mapping of the head orientation enabled the participants to have an immersive visual exploration of the virtual environment. The BP's avatar and the PP, represented by a second virtual surrogate, were seated facing each other around a square table, above which the virtual gaming board was placed. Therefore, BP could see both the own and PP avatar as well as the board and the gaming actions. Animation of the avatars' hands was performed using the inverse-kinematic algorithm provided by the HALCA library, generating convincing trajectories of the limbs during point, pick and place actions that target the boardgame tokens.

Robotic Scenario. Video feedback was streamed in real-time from the robot's cameras and viewed in stereoscopy by the BP. This allowed providing a 1PP from the robot's point of view to the BP. The robot (Fig. 3), which was located at the Chair of Automatic Control Engineering, (Munich, Germany), received goal-oriented commands (i.e., the corresponding number of the token) and planned the necessary arm trajectories to reach the respective tokens using a trapezoidal velocity profile to interpolate between the start and target poses. Similarly to the virtual scenario, the BP's head movements were mapped to the robot's head with the help of the head tracking data received from the $\mathrm{BP}$ side (Rome, Italy). The tokens in the robotic condition differed from their counterpart in the virtual scenario in that they were represented by augmented reality (AR) markers (see Fig. 2-B) whose recognition was carried out using a Kinect (Microsoft, USA) camera mounted and fixed to the robot's chest and the ARToolKit (http://www.hitl.washington.edu/artoolkit, 2002; last access $24^{\text {th }}$ March 2016) software library.

\section{Procedure}

Participants were seated comfortably in a chair and received detailed description about the P300-based BCI, the proprioceptive stimulator and the experimental task (see Sections II-B and II-C). Before the start of the experiments, the proprioceptive device was gently placed on the participant's right flexor tendon brachii with the elbow angular position of approximately $120^{\circ}$ (Fig. 1 left). A $60 \mathrm{~Hz}$ stimulation with $4.2 \mathrm{~N}$ for $30 \mathrm{~s}$ was applied. Participants were asked to pay attention to any changes in the physical and sensory perception of their arm. We took care not to influence the subjects regarding the possible illusory movements they might experience. This calibration procedure helped us to find the optimal position that helps to elicit a vivid illusory movement sensation related to the proprioceptive stimulation. All participants reported the feeling that the arm was moving downward except for Pt3 (see Table I).

After this initial calibration step, two EEG sessions were acquired during P300 copy spelling mode aiming at learning and assessing the P300-based BCI system. Subjects were asked in these sessions to focus their attention on different predefined targets, which were cued to them before the start of flashing. Each row/column of the $(3 \times 3)$ board was randomly flashed exactly 10 times. During the training sessions, subjects copyspelled seven targets, which provided labelled epochs as target or non-target trials that were then used to train an LDA classifier. The entire training procedure lasted about $6 \mathrm{~min}$. In the assessment phase, we evaluated the accuracy of the learned LDA classifier for each participant by performing 12 selections in copy spelling mode. Accuracy in this assessment phase was computed as the ratio of the number of correct selections to the total number of selections (i.e., 12; see Table III for Study 1 and Table IV for Study 2).

Then, participants played the cooperative game within the virtual (Study 1-2) and robotic scenarios in Study 2 (see Section II-C). Before the start of the game, subjects were invited to explore the environment and to verbally report what they were seeing ( $100 \mathrm{~s}$ familiarization procedure; [33]). At the end of each condition, participants verbally answered a set of questions (co-presence, embodiment, sense of control, illusionary movement, BCI interface; see Table II) assessing their UE; questions were randomly administered and partly adapted from previous studies [34], [35].

\section{STUDY 1}

Participants were immersed into a virtual environment and completed the cooperative game in two experimental conditions. In particular, tendon vibration was provided either to the biceps brachii (MovI+) or to the nearby bone (MovI-; 
TABLE II

USER EXPERIENCE MEAN + sem Answers: Copresence (Cop), EMbodiment (Emb), Sense of Control (Ctrl), Illusory Movement (iMov) AND BCI INTERFACE (Bci)

\begin{tabular}{|c|c|c|c|c|c|c|c|c|c|c|c|c|c|c|c|}
\hline \multirow{3}{*}{\multicolumn{2}{|c|}{ Items }} & \multicolumn{4}{|c|}{ Study 1} & \multicolumn{4}{|c|}{$\begin{array}{c}\text { Study } 2 \\
\text { (Healthy participants) }\end{array}$} & \multicolumn{6}{|c|}{$\begin{array}{c}\text { Study } 2 \\
\text { (People living with SCI) }\end{array}$} \\
\hline & & \multirow{2}{*}{ MovI+ } & \multirow{2}{*}{ MovI- } & \multirow{2}{*}{$\mathbf{z}$} & \multirow{2}{*}{$\mathbf{p}$} & \multirow{2}{*}{ Virtual } & \multirow{2}{*}{ Robot } & \multirow{2}{*}{$\mathbf{z}$} & \multirow{2}{*}{$\mathbf{p}$} & \multicolumn{3}{|c|}{ Virtual } & \multicolumn{3}{|c|}{ Robot } \\
\hline & & & & & & & & & & Pt1 & Pt2 & Pt3 & Pt1 & $P t 2$ & Pt3 \\
\hline Cop1 & $\begin{array}{l}\text { I had the feeling to be in the same } \\
\text { room with the other person / avatar }\end{array}$ & $52.50 \pm 4.12$ & $60.63 \pm 5.55$ & 1.36 & 0.17 & $44.70 \pm 11.92$ & $40.00 \pm 7.26$ & 0.35 & 0.72 & 30 & 30 & 40 & 40 & n.a. & 20 \\
\hline Cop2 & $\begin{array}{l}\text { I had the feeling to work directly } \\
\text { with the other person / avatar, as if } \\
\text { the technology that allowed the } \\
\text { interaction vanished }\end{array}$ & $66.25 \pm 6.80$ & $68.13 \pm 6.26$ & 0.53 & 0.59 & $48.00 \pm 10.12$ & $53.00 \pm 10.55$ & 0.25 & 0.80 & 40 & 50 & 50 & 40 & n.a. & 20 \\
\hline$E m b 1$ & $\begin{array}{l}\text { It was as if the robotic / virtual body } \\
\text { was my body }\end{array}$ & $42.50 \pm 5.90$ & $51.25 \pm 8.33$ & 1.28 & 0.20 & $36.00 \pm 9.33$ & $36.50 \pm 8.03$ & 0.07 & 0.94 & 20 & 20 & 10 & 30 & n.a. & 0 \\
\hline$E m b 2$ & $\begin{array}{c}\text { It was as if I had two bodies (here } \\
\text { and there) }\end{array}$ & $58.75 \pm 8.75$ & $59.38 \pm 7.93$ & 0.42 & 0.67 & $33.50 \pm 8.69$ & $33.00 \pm 10.41$ & 0.13 & 0.89 & 60 & 10 & 10 & 30 & n.a. & 50 \\
\hline Ctrl & $\begin{array}{c}\text { Ifelt in control of robot's / avatar's } \\
\text { actions }\end{array}$ & $71.25 \pm 9.34$ & $71.25 \pm 6.39$ & 0.00 & 1.00 & $78.30 \pm 6.34$ & $80.30 \pm 5.42$ & 0.10 & 0.92 & 60 & 80 & 50 & 40 & n.a. & 80 \\
\hline$i M o v$ & $\begin{array}{l}\text { When the robot's / avatar's arm } \\
\text { moved downward it was as if my real } \\
\text { arm moved downward }\end{array}$ & $37.50 \pm 5.26$ & $45.63 \pm 6.08$ & 1.08 & 0.28 & $40.50 \pm 11.61$ & $52.50 \pm 9.81$ & 0.83 & 0.41 & 20 & 30 & 15 & 40 & n.a. & 0 \\
\hline Bcil & $\begin{array}{l}\text { It was easy commanding through the } \\
\text { BCI interface }\end{array}$ & $75.00 \pm 8.02$ & $77.50 \pm 11.61$ & 0.10 & 0.92 & $79.90 \pm 8.41$ & $73.00 \pm 10.00$ & 0.70 & 0.48 & 40 & 80 & 70 & 50 & n.a. & 70 \\
\hline Bci2 & $\begin{array}{l}\text { It was hard looking at the flashing } \\
\text { stimuli }\end{array}$ & $16.88 \pm 4.90$ & $13.75 \pm 4.98$ & 0.55 & 0.58 & $22.50 \pm 8.47$ & $21.50 \pm 8.63$ & 0.31 & 0.75 & 30 & 0 & 20 & 30 & n.a. & 10 \\
\hline
\end{tabular}

see Section II-B) in counterbalanced order across subjects. In this way, we assessed the role of illusory movement perception over UE and performance and controlled for the effect of the vibration [29]. The stimulation in either condition (MovI+ or MovI-) started on the basis of the real-time right hand position of the virtual BP surrogate.

\section{A. Performance Measurement and Data Handling}

Participants answered on a 100-point scale ranging from 0 ("I totally disagree") to 100 ("I totally agree") for each UE's item (see Table II). Task accuracy (the ratio of correct trials to the total number of trials), OCs (number of automatic BCI-selections that follow the selection of unavailable tokens) and ITR are reported in Table III.

The ITR (bit $/ \mathrm{min}$ ) is calculated as $B / T$, where $B$ is the bit rate (bit/selection) and $T$ is the time required to make a single BCI selection. The bit rate was calculated according to the following formula:

$$
\begin{aligned}
B= & P \log _{2}(P)+P_{c} \log _{2}\left(\frac{P_{c}}{M-1}\right) \\
& -\left(1-P_{e}\right) \log _{2}\left(\frac{1-P_{e}}{M}\right)
\end{aligned}
$$

where $M=9$ is the total number of possible selections, $P+P_{e}+P_{c}=1, P$ is the probability of correct detection, $P_{c}$ is the crossover probability (i.e., probability of wrongly detecting the desired cell with other undesired available cells) and $P_{e}$ is the probability of erasure (i.e., probability of wrongly detecting the desired selection as an empty or erased cell). The new formula deviates from the one proposed by Wolpaw et al. [36] due to the incorporation of the erased selections. Note that when $P_{e}=0$, the formula for $B$ reduces to the one in [36].

Data were not normally distributed (7 out of 22 measures were not normally distributed as confirmed by
TABLE III

Performance Measures During BCI Assessment and

\begin{tabular}{|c|c|c|c|c|c|c|c|}
\hline \multirow{2}{*}{\multicolumn{2}{|c|}{$\begin{array}{c}\text { Assessment } \\
\text { Phase } \\
\text { Accuracy }\end{array}$}} & \multicolumn{2}{|c|}{ Task Accuracy } & \multicolumn{2}{|c|}{$\begin{array}{c}\text { Optimization } \\
\text { Calls }\end{array}$} & \multicolumn{2}{|c|}{ ITR } \\
\hline & & \multirow{2}{*}{$\begin{array}{c}\text { MovI+ } \\
75 \% \\
(9 / 12)\end{array}$} & \multirow{2}{*}{$\begin{array}{c}\text { MovI- } \\
83.3 \% \\
(10 / 12)\end{array}$} & \multirow{2}{*}{$\begin{array}{c}\text { MovI+ } \\
3\end{array}$} & \multirow{2}{*}{$\frac{\text { MovI- }}{4}$} & \multirow{2}{*}{$\begin{array}{r}\text { MovI+ } \\
5.771\end{array}$} & \multirow{2}{*}{$\begin{array}{r}\text { MovI- } \\
6.793\end{array}$} \\
\hline$S 1$ & $\begin{array}{c}100 \% \\
(12 / 12)\end{array}$ & & & & & & \\
\hline$S 2$ & $\begin{array}{c}100 \% \\
(12 / 12)\end{array}$ & $\begin{array}{c}100 \% \\
(12 / 12)\end{array}$ & $\begin{array}{l}91.6 \% \\
(11 / 12)\end{array}$ & 0 & 0 & 14.215 & 11.238 \\
\hline$S 3$ & $\begin{array}{l}100 \% \\
(12 / 12)\end{array}$ & $\begin{array}{c}100 \% \\
(12 / 12)\end{array}$ & $\begin{array}{l}91.6 \% \\
(11 / 12)\end{array}$ & 0 & 3 & 14.215 & 8.991 \\
\hline$S 4$ & $\begin{array}{c}100 \% \\
(12 / 12)\end{array}$ & $\begin{array}{c}100 \% \\
(12 / 12)\end{array}$ & $\begin{array}{c}100 \% \\
(12 / 12)\end{array}$ & 1 & 0 & 13.121 & 14.215 \\
\hline$S 5$ & $\begin{array}{c}100 \% \\
(12 / 12)\end{array}$ & $\begin{array}{c}62.5 \% \\
(5 / 8)\end{array}$ & $\begin{array}{l}25 \% \\
(2 / 8)\end{array}$ & 9 & 9 & 2.301 & 0.229 \\
\hline S6 & $\begin{array}{c}100 \% \\
(12 / 12)\end{array}$ & $\begin{array}{c}100 \% \\
(12 / 12)\end{array}$ & $\begin{array}{c}100 \% \\
(12 / 12)\end{array}$ & 1 & 1 & 13.121 & 13.121 \\
\hline$S 7$ & $\begin{array}{l}91.6 \% \\
(11 / 12)\end{array}$ & $\begin{array}{l}83.3 \% \\
(10 / 12)\end{array}$ & $\begin{array}{l}83.3 \% \\
(10 / 12)\end{array}$ & 1 & 0 & 8.361 & 9.058 \\
\hline 58 & $\begin{array}{c}100 \% \\
(12 / 12) \\
\end{array}$ & $\begin{array}{l}91.6 \% \\
(11 / 12) \\
\end{array}$ & $\begin{array}{l}91.6 \% \\
(11 / 12) \\
\end{array}$ & 2 & 2 & 9.633 & 9.633 \\
\hline & 98.96 & 89.06 & 83.33 & 2.13 & 2.38 & 10.09 & 9.16 \\
\hline$\stackrel{ \pm}{ \pm}$ & \pm & \pm & \pm & \pm & \pm & \pm & \pm \\
\hline & 1.04 & 5.04 & 8.63 & 1.04 & 1.08 & 1.55 & 1.53 \\
\hline
\end{tabular}
VIRTUAL INTERACTIONS (Movl+, MOVI-) IN STUDY 1

a Shapiro-Wilk test: $\mathrm{p}<0.05)$. Task accuracy, OCs, ITR and UE items were compared between the MovI+ and MovIexperimental conditions by using the non-parametric paired Wilcoxon signed rank tests. Alpha level was set to 0.05. Subject 5 completed only eight trials for each experimental condition.

\section{B. Results}

Task Accuracy and OCs. No significant differences $(\mathrm{z}=1.09, \mathrm{p}=0.27, \mathrm{r}=0.27)$ were found between the MovI + (mean \pm s.e.m., $89.06 \pm 5.04$ ) and MovI- stimulation 
$(83.33 \pm 8.63)$. OCs intervened the same number of times in the two conditions and non-significant differences $(\mathrm{z}=0.36$, $\mathrm{p}=0.71, \mathrm{r}=0.09$ ) were found between the MovI+ (mean \pm s.e.m., $2.13 \pm 1.04)$ and the MovI- stimulation $(2.38 \pm$ 1.08).

ITR. No significant differences $(\mathrm{z}=0.94, \mathrm{p}=0.34$, $\mathrm{r}=0.24)$ were found between the MovI+ $(10.09 \pm 1.55)$ and MovI- stimulation (9.16 \pm 1.53$)$.

Subjective Experience. Comparisons revealed no significant differences in UE between the MovI + and MovI- conditions (all $\mathrm{z}<1.36, \mathrm{p}>0.17, \mathrm{r}<0.34$; see Table II for means and comparisons). Participants experienced a good level of copresence (Cop1 and Cop2). The feeling of owning the virtual body (Emb1) and the sensation of having two bodies (Emb2) did not significantly differ between the proprioceptive stimulation conditions. Importantly, participants experienced a high level of being in control of the virtual agent $(\mathrm{Ctrl})$ and considered the BCI-interface easy to use (Bci1) with no fatigue for the flashing stimuli (Bci2). Relevant to the objectives of the study, participants reported weak illusory movements in the two stimulation conditions (iMov; see General Discussion).

\section{Discussion Study 1}

Study 1 assessed the participants' ability to control a virtual surrogate and the subjective user experience during virtual social interactions. We observed that participants successfully completed the virtual interactions using the P300-based BCI application and no significant differences between the two proprioceptive stimulation conditions were observed. OCs and ITR did not significantly differ between the two conditions. Importantly, participants reported a good level of control throughout the task and we observed that the different proprioceptive stimulations did not produce significantly different levels of illusory experiences in terms of embodiment, copresence and illusory movement (see General Discussion).

\section{STUDY 2}

In Study 2, we compared a group of healthy people and three participants living with SCI immersed within a virtual and robotic scenario. It has been reported that people with a SCI can experience illusory sensation of movement [37] when a vibration is applied to the tendon biceps brachii. Data from Study 1 indicated that different proprioceptive stimulations did not affect the perceived vividness of the illusory movement. Thus, in this study we compared a group of healthy people and three participants with SCI immersed within a virtual and robotic scenario using congruent only (MovI+) proprioceptive stimulation.

We tested whether people with reduced mobility due to spinal cord lesions perform similarly to healthy people [38], [39]. Moreover, we assessed if different subjective reports could be obtained when participants and people living with SCI are immersed in robotic and virtual scenarios.

\section{A. Procedure, Measures and Data Handling}

Procedure and measures were the same as in Study 1. The proprioceptive stimulation in either scenario (virtual or
TABLE IV

Performance Measures During BCI Assessment and VIRTUAL (VR)-ROBOT (RBT) INTERACTIONS IN STUDY 2

\begin{tabular}{|c|c|c|c|c|c|c|c|}
\hline \multirow{2}{*}{\multicolumn{2}{|c|}{$\begin{array}{l}\text { Assessment } \\
\text { Phase } \\
\text { Accuracy }\end{array}$}} & \multicolumn{2}{|c|}{ Task Accuracy } & \multicolumn{2}{|c|}{$\begin{array}{l}\text { Optimization } \\
\text { Calls }\end{array}$} & \multicolumn{2}{|c|}{ ITR } \\
\hline & & $V R$ & $R B T$ & $V R$ & $R B T$ & $V R$ & $R B T$ \\
\hline$S 1$ & $\begin{array}{c}100 \% \\
(12 / 12)\end{array}$ & $\begin{array}{c}100 \% \\
(12 / 12)\end{array}$ & $\begin{array}{c}100 \% \\
(12 / 12)\end{array}$ & 0 & 0 & 14.215 & 14.215 \\
\hline$S 2$ & $\begin{array}{l}83.3 \% \\
(10 / 12)\end{array}$ & $\begin{array}{l}83.3 \% \\
(10 / 12)\end{array}$ & $\begin{array}{l}91.6 \% \\
(11 / 12)\end{array}$ & 0 & 0 & 9.058 & 11.238 \\
\hline$S 3$ & $\begin{array}{c}100 \% \\
(12 / 12)\end{array}$ & $\begin{array}{c}100 \% \\
(12 / 12)\end{array}$ & $\begin{array}{c}100 \% \\
(12 / 12)\end{array}$ & 0 & 0 & 14.215 & 14.215 \\
\hline$S 4$ & $\begin{array}{c}100 \% \\
(12 / 12)\end{array}$ & $\begin{array}{c}100 \% \\
(12 / 12)\end{array}$ & $\begin{array}{l}91.6 \% \\
(11 / 12)\end{array}$ & 0 & 0 & 14.215 & 11.238 \\
\hline$S 5$ & $\begin{array}{l}83.3 \% \\
(10 / 12)\end{array}$ & $\begin{array}{l}91.6 \% \\
(11 / 12)\end{array}$ & $\begin{array}{c}100 \% \\
(12 / 12)\end{array}$ & 1 & 0 & 10.374 & 14.215 \\
\hline S6 & $\begin{array}{c}100 \% \\
(12 / 12)\end{array}$ & $\begin{array}{l}91.6 \% \\
(11 / 12)\end{array}$ & $\begin{array}{c}87.5 \% \\
(7 / 8)\end{array}$ & 1 & 0 & 10.374 & 10.096 \\
\hline$S 7$ & $\begin{array}{c}100 \% \\
(12 / 12)\end{array}$ & $\begin{array}{l}91.6 \% \\
(11 / 12)\end{array}$ & $\begin{array}{l}81.8 \% \\
(9 / 11)\end{array}$ & 3 & 6 & 8.991 & 5.630 \\
\hline$S 8$ & $\begin{array}{l}91.6 \% \\
(11 / 12)\end{array}$ & $\begin{array}{l}91.6 \% \\
(11 / 12)\end{array}$ & $\begin{array}{c}83.3 \% \\
(10 / 12)\end{array}$ & 0 & 0 & 11.238 & 9.058 \\
\hline$s 9$ & $\begin{array}{l}91.6 \% \\
(11 / 12)\end{array}$ & $\begin{array}{c}100 \% \\
(12 / 12)\end{array}$ & $\begin{array}{c}100 \% \\
(12 / 12)\end{array}$ & 2 & 1 & 12.184 & 13.121 \\
\hline S10 & $\begin{array}{l}91.6 \% \\
(11 / 12)\end{array}$ & $\begin{array}{c}100 \% \\
(12 / 12)\end{array}$ & $\begin{array}{c}100 \% \\
(12 / 12)\end{array}$ & 0 & 1 & 14.215 & 13.121 \\
\hline $\begin{array}{c}\text { Mean } \\
\pm \\
\text { s.e.m. }\end{array}$ & $\begin{array}{l}94.17 \\
\pm \\
2.17\end{array}$ & $\begin{array}{c}95 \\
\pm \\
1.84\end{array}$ & $\begin{array}{l}93.75 \\
\pm \\
2.26\end{array}$ & $\begin{array}{c}0.7 \\
\pm \\
0.33\end{array}$ & $\begin{array}{c}0.8 \\
\pm \\
0.59\end{array}$ & $\begin{array}{c}11.91 \\
\pm \\
0.69\end{array}$ & $\begin{array}{c}11.61 \\
\pm \\
0.88\end{array}$ \\
\hline$P t 1$ & $\begin{array}{c}41.6 \% \\
(5 / 12) \\
83.3 \% \\
(5 / 6)\end{array}$ & $\begin{array}{l}75 \% \\
(9 / 12)\end{array}$ & $\begin{array}{l}33.3 \% \\
(4 / 12)\end{array}$ & 5 & 11 & 5.092 & 0.589 \\
\hline Pt2 & $\begin{array}{c}100 \% \\
(12 / 12)\end{array}$ & $\begin{array}{c}91.6 \% \\
(11 / 12)\end{array}$ & n.a. & 1 & n.a. & 10.374 & n.a. \\
\hline Pt3 & $\begin{array}{c}100 \% \\
(12 / 12)\end{array}$ & $\begin{array}{l}66.6 \% \\
(8 / 12)\end{array}$ & $\begin{array}{c}100 \% \\
(12 / 12)\end{array}$ & 2 & 0 & 4.811 & 14.215 \\
\hline
\end{tabular}

robotic) started on the basis of the real-time virtual/robotic surrogate's right hand position. While for the virtual avatar all position data could be accessed locally, data of the endeffector position for the robotic avatar were sent remotely over the Internet to the host PC.

Due to encountered technical failure, Subject 7 and Subject 8 completed only 11 and 8 trials respectively in the robotic scenario. Within the SCI group Pt1 required two P300 training sessions and Pt2 decided to complete only the virtual scenario (see Table IV).

Data were not normally distributed (10 out of 22 measures were not normally distributed as confirmed by a Shapiro-Wilk test: $\mathrm{p}<0.05$ ). Task accuracy, OCs, ITR and answers to the UE questionnaire were compared between the virtual and robotic scenarios using non-parametric paired Wilcoxon signed rank tests for healthy participants (see Table IV for accuracies, OCs and ITR; see Table II for UE answers). Alpha level was set at 0.05 .

Moreover, performance and subjective measures of each individual patient underwent two planned comparisons using case-control statistics according to Crawford analysis [40] (see Table S1 in the supplementary material for task accuracy, OCs and ITR; see Table S2 for UE). Alpha level was thus corrected to 0.025 and better controlled for small size of the control group and for violations from normality [41]. 


\section{B. Results: Healthy Participants}

Task accuracy and OCs. We found no significant differences in the accuracy levels $(\mathrm{z}=0.73, \mathrm{p}=0.46, \mathrm{r}=0.16)$ between the virtual (mean \pm s.e.m., $93.94 \pm 1.98$ ) and robotic scenario $(93.60 \pm 2.34)$. Similarly, the number of OCs did not significantly differ $(\mathrm{z}=0.00, \mathrm{p}=1.00, \mathrm{r}=0.00)$ between the virtual (mean \pm s.e.m., $0.70 \pm 0.33$ ) and robotic condition $(0.80 \pm 0.59)$.

ITR. No significant differences $(\mathrm{z}=0.49, \mathrm{p}=0.62$, $\mathrm{r}=0.11)$ were found between the virtual $(11.91 \pm 0.69)$ and robotic scenario (11.92 \pm 0.73$)$.

Subjective Experience. The analysis revealed no significant differences in UE between the virtual and robotic scenarios (all $\mathrm{z}<0.83, \mathrm{p}>0.41, \mathrm{r}<0.20$ ). Importantly participants experienced a high level of being in control of the virtual agent (Ctrl) and considered the BCI-interface easy to use (Bci1) with no fatigue for the flashing stimuli (Bci2).

\section{Results: People With SCI}

Patient1. Both in the virtual and the robotic scenarios Pt1 had lower task accuracy levels, higher number of OCs and lower ITR relative to the control group (all $\mathrm{p}<0.022$; see Table S1). Moreover, a trend suggested that Pt1 had reduced feeling of being in control of the robot relative to healthy controls $(t=-2.24, \mathrm{p}=0.052$; see Table $\mathrm{S} 2)$.

Patient 2 . Pt 2 performed only within the virtual scenario, where task accuracy, OCs and ITR did not significantly differ relative to controls (see Table S1). No significant differences were found in the subjective evaluation of the virtual interaction (see Table S2).

Patient3. While Pt 3 performance did not significantly differ relative to controls in the robot scenario, task accuracy and ITR in the virtual interaction were lower relative to controls $(\mathrm{t}<-3.09, \mathrm{p}<0.01$; see Table $\mathrm{S} 1$ in the supplementary material).

Although our SCI sample is limited, subjective reports might suggest a role of the lesion level and accuracy for illusory feelings (copresence, embodiment and illusory movement perception). The low feeling of being in control (Ctrl) and the reduced comfort of the BCI (Bci1, Bci2) in Pt1 might have been affected by the low performance in the robotic condition and by the relatively high number of OCs in both scenarios. Conversely, Pt2 achieved high accuracy and reported higher ratings in evaluating the $\mathrm{BCI}$ comfort. Finally, Pt3 had the highest lesion level relative to the other SCI patients and reported the lowest ratings for the illusory movement, copresence and embodiment feelings relative to Pt1 and Pt2. Importantly, Pt3 did not report the illusory movement in the initial proprioceptive assessment (see Section II-D). However, we did not find any significant difference between healthy and SCI people in body illusions experience (see Table S2).

\section{Discussion Study 2}

We observed that participants successfully accomplished the cooperative interaction using a P300-based BCI. Similar to Study 1 , OCs allowed to preserve high task accuracy levels throughout the task and may have contributed to high levels of perceived control (Ctrl). The illusory experience in terms of embodiment, copresence and illusory movement did not significantly differ between the virtual and the robotic scenario (see Section V). Moreover, we found no significant differences in terms of task accuracy, OCs and ITR between the two experimental conditions.

The performance of participants with a spinal cord injury, however, was more variable with respect to controls. Pt1 was able to complete the assigned tasks with a higher number of OCs and lower levels of accuracy relative to controls and reported a reduced feeling of control during the robotic scenario. Moreover, while Pt2 had a good level of task accuracy that did not differ from healthy participants, Pt3 had a lower level of accuracy in the virtual interaction relative to controls.

Overall, we observed that healthy participants were able to use the developed system and confirmed the relevance of the optimization module to reduce the number of unwanted actions (see S7 in Table IV). Importantly, people living with SCI reported subjective experience that did not differ relative to controls but had a more variable performance in the control of the virtual avatar and the robotic surrogate.

\section{General Discussion}

We combined virtual reality, robotics and BCI technologies with current knowledge on body-ownership illusions into a single framework, and evaluated the resulting system with healthy and people with SCI in both local and remote social interactions. In particular, we assessed the contribution of proprioceptive feedback on embodiment feelings in both healthy and a small group of SCI patients and explored whether the type of the immersive environment (i.e., virtual or physical) might influence performance measures during a social interaction.

We found no effect in performance and embodiment feelings following proprioceptive stimulation (Study 1) and we did not observe dissimilar performance in both the virtual and robotic scenarios (Study 2). Importantly, the patients' performance and subjective experience were not significantly different to those found in the healthy group. However, it has to be noted that task accuracy, OCs and ITR appeared susceptible to higher variability in people with SCI.

\section{A. Performance Measures and Illusory Movement}

In this work, we tried to investigate the contribution of proprioceptive stimulation, delivered when the virtual or robotic arm was pointing to the selected token, to illusory movement and body-ownership feelings over a virtual and robotic surrogate. We observed that different proprioceptive stimulations (Study 1) and immersive environments (Study 2) did not influence the illusory movement perception and participants reported low movement illusion relative to previous reports [28], [29]. Previous studies used monitor-based stimulation in P300 systems and showed no significant difference in BCI use in people with spinal cord injury [38], [39] relative to controls. Although we used an immersive setup in this work, the observed variability in the use of the $\mathrm{BCI}$ device in healthy 
as well as in people living with SCI cannot be accounted for by difficulty in using the BCI within the HMD in general. The fact that all participants performed correctly the BCI assessment phase (see Table III for Study 1 and Table IV for Study 2) might suggest that $2 \mathrm{D}$ scenarios are easier to complete and might be less distracting in terms of visual attention.

In the present work, participants performed a P300-based task and were stimulated with a force of $4.2 \mathrm{~N}$ at $60 \mathrm{~Hz}$. Crucially, the fact that participants did not rate the illusion in the preliminary assessment (see Section II-D), and that participants estimated the overall illusory movement perception at the end of each experimental condition, limits the possibility to compare the proprioceptive and visuo-proprioceptive contribution to illusory movements (i.e., comparing the illusion during the proprioceptive assessment phase with the social BCI-based interactions).

Our results extend previous findings that observed improvements in control using a motor-imagery-based BCI [27], [42], [43] when proprioceptive stimulation was matched by visual feedback [27], [42], [43], [44] in a group of healthy participants. We found a relatively low level of bodily illusions and a high level of task accuracy across the studies. Moreover, possible mismatches between visual feedback of a moving limb and the proprioceptive stimulation may have affected illusory movement experiences [22], [45].

We do not exclude that the unfamiliar setting of the experienced social interaction (people had to remain in a fixed posture throughout the entire testing sessions while interacting with a virtual or real person) may have limited the possibility that participants experienced deeper embodiment feelings. Combined with the small sample size of our study, this may have consequently limited our ability to draw stronger conclusions.

\section{B. Body-Ownership Over BCl-Controlled Surrogates}

Body-ownership over external objects has been studied with rubber hands [46], virtual and real bodies [34], [47] and mannequins [48]. Congruent visual feedback of intended actions is important for generating an illusory feeling of owning and controlling an external object [49], [50]. The sense of agency is indeed a fundamental component of embodiment processes [51] and is influenced by sensorimotor congruencies among the executed action, its sensorimotor re-afferences [52] and the feedback about goal achievement [53], [54].

Participants were able to use the BCI (as assessed in the BCI-assessment phase, see Table III for Study 1 and Table IV for Study 2). In order to improve task accuracy (i.e., reduce the number of erroneous selections of empty cells) we allowed the software to intervene with automatic error detection and BCI-selection repetition. Such optimization process might have played an important role in maintaining the sensation of being in control of the virtual and robotic surrogate. Nonetheless, participants reported a relatively low sensation of owning the virtual and robotic body. Surprisingly we observed a low level of embodiment in Pt3, who did not perceive the illusory movement sensation (see Procedure and Table I) and in previous studies experienced high level of ownership over a static rubber hand [23]. The fact that embodiment and illusory movement feelings did not change between the virtual and robotic condition might indirectly hint that delays between BCI output and the start of the reaching movement in the virtual and robotic conditions were not a crucial factor for embodiment sensations in this particular experimental setup. Note that in our present studies, we did not apply visuo-tactile stimuli over participants' hands to elicit illusory ownership sensations over the virtual and robotic bodies [19], but rather we tried to achieve good levels of perceived control and body-ownership by the implementation of OCs and through congruent proprioceptive stimulation respectively. Crucially, participants were not required to make any overt movement throughout the task and the only congruency between a motor action and its visuo-proprioceptive consequence was related to participants' head rotations and the corresponding changes in the field of view. That is, whenever participants moved their head, a congruent perspective change followed their movements [11], [55].

Finally, we cannot exclude that tendon stimulation might have induced the sensation of a passive extension rather than a voluntary action, since no real movements and no motor imagery was asked to be performed by subjects during the stimulation. However, participants reported a good level of perceived control over the observed actions. On the other hand, low scores on perceived ownership point to the need for ameliorating the integration of visual [45] and motor sensory [56] feedback through external stimulation in order to elicit an active feeling of controlling an agent's body observed from a first person perspective.

\section{CONCLUSION}

Previous studies showed a possible role of embodiment [15], [16], [17] and proprioceptive feedback [27], [42], [43] in altering motor-imagery based EEG signals that can be used for BCI control. On this account, we performed two studies and investigated in a group of healthy participants and a small group of people living with SCI the ability to use a P300-based BCI system within immersive scenarios, while proprioceptive feedback was provided aiming at increasing the level of embodiment into a virtual and robotic surrogate.

In Study 1, we found high accuracy and no significant difference in BCI control and embodiment feelings toward the virtual surrogate using congruent and incongruent stimulation. In Study 2, healthy participants did not significantly differ between the immersive robotic and virtual scenario and patients appeared to control the virtual and robotic surrogate with good performance levels, despite some observed differences (as indexed by Crawford analyses).

Our results hint at possible directions that can be beneficial in improving the development of immersive systems with realistic sensory feedback (e.g., tactile stimulation supporting visual feedback to ameliorate embodiment sensations), and integrating optimization modules to reduce the rate of committed errors. Further studies are necessary to investigate the use of alternative P300 stimulations and classification [57], [58] within immersive systems [59] in order to reduce the number of software interventions and to improve the use of BCI systems. 
Overall, we reported two cross-disciplinary studies integrating knowledge from different research fields namely, 1) body ownership over virtual and robotic devices, 2) illusory movement following tendon vibration, 3) virtual and 4) robotic systems coupled with 5) BCI technology. This integration resulted in a system that provided a local-virtual and a remoterobotic social interaction to BCI users. The integration of engineering and neuroscience opens the gate to promising future applications, aiming at controlling external devices through brain signals with both high performance levels and enhanced feelings of owning the controlled device. Further studies are yet necessary to test whether user's sensorimotor experience may enhance the ability of people in using BCI technology.

\section{REFERENCES}

[1] T. Aflalo et al., "Decoding motor imagery from the posterior parietal cortex of a tetraplegic human," Science, vol. 348, no. 6237, pp. 906-910, May 2015.

[2] L. R. Hochberg et al., "Reach and grasp by people with tetraplegia using a neurally controlled robotic arm," Nature, vol. 485, no. 7398, pp. 372-375, 2012.

[3] R. Leeb, D. Friedman, G. R. Müller-Putz, R. Scherer, M. Slater, and G. Pfurtscheller, "Self-paced (asynchronous) BCI control of a wheelchair in virtual environments: A case study with a tetraplegic," Comput. Intell. Neurosci., vol. 2007, Jan. 2007, Art. no. 7.

[4] J. D. R. Millán et al., "Combining brain-computer interfaces and assistive technologies: State-of-the-art and challenges," Front. Neurosci., vol. 4, no. 161, pp. 1-15, Jan. 2010

[5] C. Brunner et al., "BNCI horizon 2020: Towards a roadmap for the BCI community," Brain-Comput. Interfaces, vol. 2, no. 1, pp. 1-10, Jan. 2015.

[6] A. Steed et al., "Beaming: An asymmetric telepresence system," IEEE Comput. Graph. Appl., vol. 32, no. 6, pp. 10-17, Nov. 2012.

[7] J. Mattout, "Brain-computer interfaces: A neuroscience paradigm of social interaction? A matter of perspective," Front. Human Neurosci., vol. 6, p. 114, Jan. 2012.

[8] R. Scherer and G. Pfurtscheller, "Thought-based interaction with the physical world," Trends Cognit. Sci., vol. 17, no. 10, pp. 490-492, Oct. 2013.

[9] B. Lenggenhager, T. Tadi, T. Metzinger, and O. Blanke, "Video ergo sum: Manipulating bodily self-consciousness," Science, vol. 317 no. 5841, pp. 1096-1099, Aug. 2007.

[10] M. V. Sanchez-Vives and M. Slater, "From presence to consciousness through virtual reality," Nature Rev. Neurosci., vol. 6, no. 4, pp. 332-339, Apr. 2005.

[11] M. Slater, "Place illusion and plausibility can lead to realistic behaviour in immersive virtual environments," Philos. Trans. Roy. Soc. B, Biol. Sci., vol. 364, no. 1535 , pp. 3549-3557, Dec. 2009

[12] R. Leeb, F. Lee, C. Keinrath, R. Scherer, H. Bischof, and G. Pfurtscheller, "Brain-computer communication: Motivation, aim, and impact of exploring a virtual apartment," IEEE Trans. Neural Syst. Rehabil. Eng., vol. 15, no. 4, pp. 473-482, Dec. 2007.

[13] F. Nijboer, N. Birbaumer, and A. Kübler, "The influence of psychological state and motivation on brain-computer interface performance in patients with amyotrophic lateral sclerosis-A longitudinal study," Front. Neurosci., vol. 4, no. 55, pp. 1-13, Jan. 2010.

[14] S. C. Kleih, F. Nijboer, S. Halder, and A. Kübler, "Motivation modulates the P300 amplitude during brain-computer interface use," Clin. Neurophysiol., vol. 121, no. 7, pp. 1023-1031, Jul. 2010.

[15] M. Alimardani, S. Nishio, and H. Ishiguro, "Effect of biased feedback on motor imagery learning in BCI-teleoperation system," Front. Syst. Neurosci., vol. 8, no. 4, p. 52, Jan. 2014.

[16] E. F. Pavone, G. Tieri, G. Rizza, E. Tidoni, L. Grisoni, and S. M. Aglioti, "Embodying others in immersive virtual reality: Electro-cortical signatures of monitoring the errors in the actions of an avatar seen from a first-person perspective," J. Neurosci., vol. 36, no. 2, pp. 268-279, Jan. 2016.

[17] G. Padrao, M. Gonzalez-Franco, M. V. Sanchez-Vives, M. Slater, and A. Rodriguez-Fornells, "Violating body movement semantics: Neural signatures of self-generated and external-generated errors," Neuroimage, vol. 124, pp. 147-156, Jan. 2016
[18] O. Blanke, "Multisensory brain mechanisms of bodily selfconsciousness," Nature Rev. Neurosci., vol. 13, no. 8, pp. 556-571, Aug. 2012.

[19] M. Slater, D. Perez-Marcos, H. H. Ehrsson, and M. V. Sanchez-Vives, "Towards a digital body: The virtual arm illusion," Front. Human Neurosci., vol. 2, no. 8, p. 6, Jan. 2008

[20] D. Perez-Marcos, M. Slater, and M. V. Sanchez-Vives, "Inducing a virtual hand ownership illusion through a brain-computer interface," Neuroreport, vol. 20, no. 6, pp. 589-594, Apr. 2009.

[21] M. Alimardani, S. Nishio, and H. Ishiguro, "Humanlike robot hands controlled by brain activity arouse illusion of ownership in operators," Sci. Rep., vol. 3, p. 2396, Jan. 2013.

[22] G. M. Goodwin, D. I. McCloskey, and P. B. C. Matthews, "Proprioceptive illusions induced by muscle vibration: Contribution by muscle spindles to perception?" Science, vol. 175, no. 4028, pp. 1382-1384, Mar. 1972.

[23] E. Tidoni, L. Grisoni, M. T. Liuzza, and S. M. Aglioti, "Rubber hand illusion highlights massive visual capture and sensorimotor face-hand remapping in a tetraplegic man," Restor. Neurol. Neurosci., vol. 32, no. 5, pp. 611-622, Jul. 2014.

[24] M. Carrozzino, F. Tecchia, S. Bacinelli, C. Cappelletti, and M. Bergamasco, "Lowering the development time of multimodal interactive application," in Proc. ACM SIGCHI Int. Conf. Adv. Comput. Entertainment Technol. (ACE), 2005, pp. 270-273.

[25] F. Tecchia, "A flexible framework for wide-spectrum VR development," Presence Teleoper. Virtual Environ., vol. 19, no. 4, pp. 302-312, Aug. 2010.

[26] M. Slater et al., "Understanding and realizing presence in the Presenccia project," IEEE Comput. Graph. Appl., vol. 27, no. 4, pp. 90-93, Jul. 2007

[27] D. Leonardis, A. Frisoli, M. Solazzi, and M. Bergamasco, "Illusory perception of arm movement induced by Visuo-proprioceptive sensory stimulation and controlled by motor imagery," in Proc. IEEE Haptics Symp. (HAPTICS), 2012, pp. 421-424.

[28] E. Tidoni, G. Fusco, D. Leonardis, A. Frisoli, M. Bergamasco, and S. M. Aglioti, "Illusory movements induced by tendon vibration in rightand left-handed people," Exp. Brain Res., vol. 233, no. 2, pp. 375-383, Feb. 2015.

[29] E. Naito, H. H. Ehrsson, S. Geyer, K. Zilles, and P. E. Roland, "Illusory arm movements activate cortical motor areas: A positron emission tomography study," J. Neurosci., vol. 19, no. 14, pp. 6134-6144, Jul. 1999.

[30] C. Guger et al., "How many people are able to control a P300based brain-computer interface (BCI)?" Neurosci. Lett., vol. 462, no. 1, pp. 94-98, Oct. 2009.

[31] C. Kapeller, K. Kamada, H. Ogawa, R. Prueckl, J. Scharinger, an C. Guger, "An electrocorticographic BCI using code-based VEP for control in video applications: A single-subject study," Front. Syst. Neurosci., vol. 8, no. 8, pp. 1-8, Aug. 2014.

[32] M. Gillies and B. Spanlang, "Comparing and evaluating real time character engines for virtual environments," Presence Teleoper. Virtual Environ., vol. 19, no. 2, pp. 95-117, Apr. 2010.

[33] M. Slater, B. Spanlang, M. V. Sanchez-Vives, and O. Blanke, "First person experience of body transfer in virtual reality," PLoS ONE, vol. 5 , no. 5, p. e10564, May 2010.

[34] M. V. Sanchez-Vives, B. Spanlang, A. Frisoli, M. Bergamasco, and M. Slater, "Virtual hand illusion induced by visuomotor correlations," PLoS ONE, vol. 5, no. 4, p. e10381, Jan. 2010.

[35] D. Friedman, R. Pizarro, K. Or-Berkers, S. Neyret, X. Pan, and M. Slater, "A method for generating an illusion of backwards time travel using immersive virtual reality-An exploratory study," Front. Psychol., vol. 5, no. 9, pp. 1-15, Sep. 2014.

[36] J. R. Wolpaw et al., "Brain-computer interface technology: A review of the first international meeting," IEEE Trans. Rehabil. Eng., vol. 8, no. 2, pp. 73-164, Jun. 2000.

[37] G. Fusco, E. Tidoni, N. Barone, C. Pilati, and S. M. Aglioti, "Illusion of arm movement evoked by tendon vibration in patients with spinal cord injury," Restorative Neurol. Neurosci., vol. 34, no. 5, pp. 815-826, Sep. 2016.

[38] F. Piccione et al., "P300-based brain computer interface: Reliability and performance in healthy and paralysed participants," Clin. Neurophysiol., vol. 117, no. 3, pp. 531-537, Mar. 2006

[39] S. Ikegami, K. Takano, N. Saeki, and K. Kansaku, "Operation of a P300based brain-computer interface by individuals with cervical spinal cord injury," Clin. Neurophysiol., vol. 122, no. 5, pp. 991-996, May 2011. 
[40] J. R. Crawford, P. H. Garthwaite, and S. Porter, "Point and interval estimates of effect sizes for the case-controls design in neuropsychology: Rationale, methods, implementations, and proposed reporting standards," Cogn. Neuropsychol., vol. 27, no. 3, pp. 245-260, May 2010.

[41] J. R. Crawford, P. H. Garthwaite, A. Azzalini, D. C. Howell, and K. R. Laws, "Testing for a deficit in single-case studies: Effects of departures from normality," Neuropsychologia, vol. 44, no. 4, pp. 666-677, 2006.

[42] A. Ramos-Murguialday et al., "Proprioceptive feedback and brain computer interface (BCI) based neuroprostheses," PLOS ONE, vol. 7, no. 10, p. e47048, Oct. 2012.

[43] L. Yao, J. Meng, X. Sheng, D. Zhang, and X. Zhu, "A novel calibration and task guidance framework for motor imagery BCI via a tendon vibration induced sensation with kinesthesia illusion," J. Neural Eng., vol. 12, no. 1, p. 016005, Feb. 2015

[44] M. D. Rinderknecht, Y. Kim, L. Santos-Carreras, H. Bleuler, and R. Gassert, "Combined tendon vibration and virtual reality for poststroke hand rehabilitation," in Proc. World Haptics Conf. (WHC), 2013, pp. 277-282.

[45] G. Tieri, E. Tidoni, E. F. Pavone, and S. M. Aglioti, "Mere observation of body discontinuity affects perceived ownership and vicarious agency over a virtual hand," Exp. Brain Res., 2015, Art. no. 17139.

[46] M. Botvinick and J. Cohen, "Rubber hands 'feel' touch that eyes see," Nature, vol. 391, no. 6669, p. 756, Feb. 1998.

[47] H. H. Ehrsson, "The experimental induction of out-of-body experiences," Science, vol. 317, no. 5841, p. 1048, Aug. 2007.

[48] V. I. Petkova and H. H. Ehrsson, "If I were you: Perceptual illusion of body swapping," PLOS ONE, vol. 3, no. 12, p. e3832, Jan. 2008.

[49] A. Kalckert and H. H. Ehrsson, "Moving a rubber hand that feels like your own: A dissociation of ownership and agency," Front. Human Neurosci., vol. 6, no. 3, p. 40, Jan. 2012.

[50] A. Kalckert and H. H. Ehrsson, "The spatial distance rule in the moving and classical rubber hand illusions," Consciousness Cognit., vol. 30, pp. 118-132, Oct. 2014.

[51] M. Tsakiris, S. Schütz-Bosbach, and S. Gallagher, "On agency and bodyownership: Phenomenological and neurocognitive reflections," Conscious. Cogn., vol. 16, no. 3, pp. 645-660, Sep. 2007.

[52] C. Farrer, N. Franck, N. Georgieff, C. Frith, J. Decety, and M. Jeannerod, "Modulating the experience of agency: A positron emission tomography study," Neuroimage, vol. 18, no. 2, pp. 324-333, Feb. 2003.

[53] D. Kumar and N. Srinivasan, "Naturalizing sense of agency with a hierarchical event-control approach," PLoS ONE, vol. 9, no. 3, p. e92431, Jan. 2014.

[54] N. Evans, S. Gale, A. Schurger, and O. Blanke, "Visual feedback dominates the sense of agency for brain-machine actions," PLOS ONE, vol. 10, no. 6, p. e0130019, 2015.

[55] A. Maselli and M. Slater, "The building blocks of the full body ownership illusion," Front. Human Neurosci., vol. 7, no. 3, p. 83, Jan. 2013.

[56] D. Leonardis, A. Frisoli, M. Barsotti, M. Carrozzino, and M. Bergamasco, "Multisensory feedback can enhance embodiment within an enriched virtual walking scenario," Presence, vol. 23, no. 3, pp. 253-266, Oct. 2014.

[57] B. Blankertz, S. Lemm, M. Treder, S. Haufe, and K.-R. Müller, "Singletrial analysis and classification of ERP components-A tutorial," Neuroimage, vol. 56, no. 2, pp. 814-825, May 2011

[58] T. Kaufmann, S. M. Schulz, C. Grünzinger, and A. Kübler, "Flashing characters with famous faces improves ERP-based brain-computer interface performance," J. Neural Eng., vol. 8, no. 5, p. 056016, Oct. 2011.

[59] I. Käthner, A. Kübler, and S. Halder, "Rapid P300 brain-computer interface communication with a head-mounted display," Front. Neurosci. vol. 9, no. 5, p. 207, 2015.

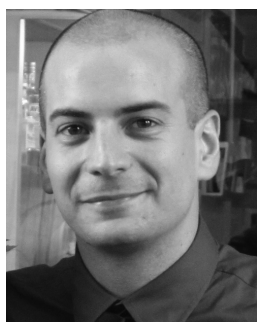

Emmanuele Tidoni received the B.S. degree in sciences of behaviour and social relations from the University of Bologna, Italy, in 2006, the M.S. degree in neuropsychology and lifelong functional Rehabilitation from University of Bologna, Italy, in 2008, and the Ph.D. degree in cognitive plasticity and rehabilitation at Sapienza University of Rome, Italy.

His research interest revolve around the multimodal assessment of embodiment with particular emphasis on ownership and agency of artificial physical-robots and virtual-characters using behavioral, virtual reality and neurophysiological approaches.

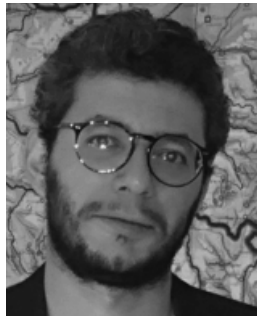

embodiment.
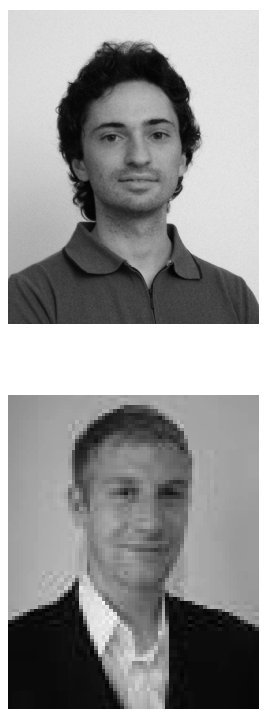

Christoph Kapeller received the B.Sc. and M.Sc. degrees in biomedical engineering from the Graz University of Technology, Graz, Austria, in 2009 and 2012, respectively. He is currently a Ph.D. degree student of computer science at the Johannes Kepler University, Linz, Austria.

$\mathrm{He}$ is currently research scientist at Guger Technologies OG in Austria. His research interests involve the real-time analysis for invasive and non-invasive brain-computer interfaces.

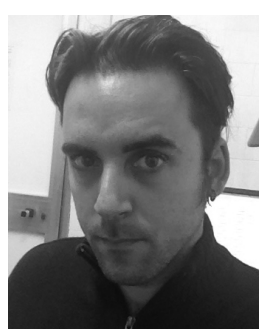

Gabriele Fusco received the B.Sc. degree and the M.Sc. both in cognitive neurosciences from the Faculty of Psychology, University of Rome "Sapienza", Rome, Italy. He is currently a Ph.D. degree student in cognitive, affective and social neurosciences (CoSAN) directed by Prof. Salvatore Maria Aglioti afferent to the Department of Psychology, University of Rome "Sapienza", Rome, Italy.

His research interests include illusory perception, spinal cord injury, brain neuromodulation and brain-computer interfaces $(\mathrm{BCl})$.

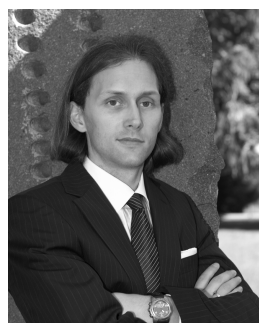

Christoph Guger studied biomedical engineering at the University of Technology Graz and Johns Hopkins University, Baltimore, MD, USA. $\mathrm{He}$ then carried out research work at the Department of Medical Informatics (Prof. Pfurtscheller) at the University of Technology Graz and received the Ph.D. degree, in 1999. The topic of his Ph.D. work was the design of an EEG-based brain-computer interface. This was the first realtime $\mathrm{BCl}$ system with continuous feedback.

$\mathrm{He}$ also developed the real-time analysis with common spatial patterns, which is still the fastest and most accurate approach for oscillatory $\mathrm{BCls}$, and also developed a $\mathrm{P} 300 \mathrm{BCl}$ with very high accuracy and speed. In recent years, he also worked with ALS and tetraplegic patients in different countries. He co-founded G.TEC 1999, and has worked there ever since. He has dozens of peer-reviewed publications, including three open access publications in Frontiers in Neuroscience that he led (and co-authored with Dr. Edlinger).

Mr. Guger was awarded the internationally recognized EY Entrepreneur Award. 


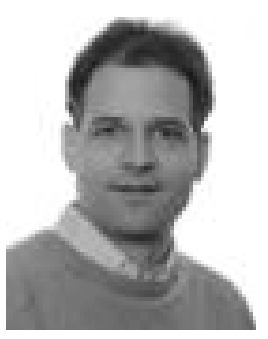

Christoph Hintermüller received the diploma in biomedical engineering at the University of Technology, Graz, in 2003, and the Ph.D. degree in medical informatics at the Institute for Biomedical Engineering (Prof. Tilg) at the Private University for Health Sciences in 2006.

After 3.5 years at the laboratory for Tomographic Microscopy and coherent radiology experiments of the Swiss Light Source at the Paul Scherrer Institute, Villigen, Switzerland, he joined g.tec in 2010 and was promoted to Chief Research Officer in 2013. His research interests are the development, optimization and evaluation of sensor systems and online bio-signal and image processing systems to noninvasively extract and image physiological, especially electrophysiological, features and processes for diagnostic purpose, rehabilitation and personal health support.

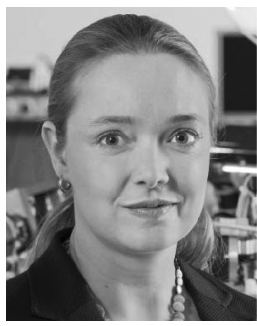

Angelika Peer received the Diploma Engineering degree in electrical engineering and information technology in 2004 and the Doctor of Engineering degree in 2008 from the same university.

She is currently Full Professor at the Bristol Robotics Laboratory, University of the West of England, Bristol, U.K. Before she was Senior Researcher and Lecturer at the Institute of Automatic Control Engineering and TUM-IAS Junior Fellow of the Institute of Advanced Studies of the Technical University of Munich, Germany. Her research interests include robotics, haptics, teleoperation, human-human and human-robot interaction as well as human motor control.

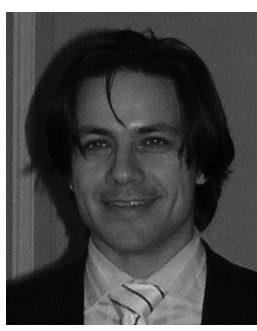

Antonio Frisoli received the M.Sc. degree in mechanical engineering, in 1998, the Ph.D. degree with honors in industrial and information engineering from Scuola Superiore Sant'Anna, Italy, in 2002.

$\mathrm{He}$ is Associate Professor of Mechanical Engineering at Scuola Superiore Sant'Anna, where he is currently head of the Human Robot Interaction (HRI) area at PERCRO laboratory TeCIP and former Chair of the IEEE Technical Committee on Haptics. His research interests concern the design and control of haptic devices and robotic systems, rehabilitation robotics, advanced $\mathrm{HRI}$ and kinematics.

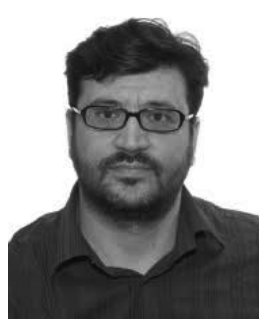

Franco Tecchia is Assistant Professor in computer science with primary specialization on software engineering and computer graphics. His research interests span the full spectrum of technology-oriented research for virtual and augmented reality: computer graphics, and computer vision, with a particular interest for the immersive visualization and augmented reality, and for the engineering aspects of complex software architectures to be employed in time-critical task, a field where he has accumulated a broad experience on the creation of complex VR systems.

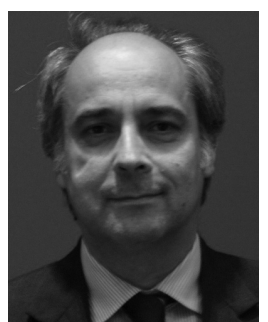

Massimo Bergamasco received the M.Sc. degree in mechanical engineering from University of Pisa, in 1985.

$\mathrm{He}$ is Full Professor of Applied Mechanics with the Faculty of Engineering, Scuola Superiore Sant'Anna, Pisa, Italy, since 2006. He is the responsible of the PERCRO Laboratory which he founded in 1994. During his research activities, he has been the responsible of many national and international projects in the field of the design environment systems. and the realization of haptic devices and virtual

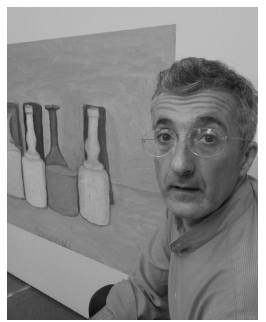

Salvatore Maria Aglioti received the M.D. degree from University of Pisa, in 1983, and the specialty in neurolog, in 1987, from the University of Verona, Italy.

His research interests revolve around the brain representations underlying corporeal awareness in healthy, brain damaged and spinal cord injured people. 